\title{
Article
}

\section{Dynamics of Single-Action Pneumatic Actuators}

\author{
Michał Bartyś \\ Institute of Automatic Control and Robotics, Warsaw University of Technology, Boboli 8, 02-525 Warsaw, Poland; \\ Michal.Bartys@pw.edu
}

\begin{abstract}
The asymmetry in the dynamics of an electro-pneumatic actuating device consisting of an electro-pneumatic transducer and a single-action pneumatic actuator was unexpectedly found experimentally. This asymmetry manifests in response to large step excitations. The dynamic asymmetry effect is understood as a change in the shape of the response of an actuator depending on the direction of the actuators stem movement. The questions appears: How to explain this effect? Does this phenomenon reflect thermodynamic air processes? Is it connected with air-to-mechanical energy conversion? Together, six working hypotheses explaining this effect were formulated. The asymmetry was studied in detail using analytical and simulation modeling, as well as experimental research. In this respect, a nonlinear analytical model was developed, tuned, and later solved using simulations. The simulation model was verified based on the experiment data. In addition, the problem of the efficiency in the energy conversion of a single-action actuator was discussed and, in result, the maximum theoretical energy efficiency was determined. Subsequently, all six working hypotheses were verified. Finally, the hypothesis explaining asymmetry as an effect of the different thermodynamic air processes in both actuator's stem travel directions was confirmed.
\end{abstract}

Keywords: fluid mechanics, effectiveness of energy conversion, single-action pneumatic actuator, analytical model, simulations, dynamics, asymmetry.

\section{Introduction}

Single-action pneumatic actuators belong to the family of final control elements, which have been applied at a large scale in industry for many years $[1,2]$. To date, sufficiently competitive counterparts for use in control valves have yet to be developed specifically in explosive zones or water steam installations used in the power, chemical, petrochemical, pharmaceutical, and food industries. A simple, robust, reliable, and rugged construction exhibiting resistance to chemical agents, vibrations, and environmental pollution at low cost has reinforced the use of pneumatic actuators in the field of control engineering.

Although there are many solutions to a single-action pneumatic actuator [3-6], in the present paper, we focus on a family of spring-and-diaphragm actuators, as illustrated in Figs. 1 and 2 with regards to energy effectiveness. Deliberations regarding energy savings in double-action pneumatic actuators can be found in [7]. 


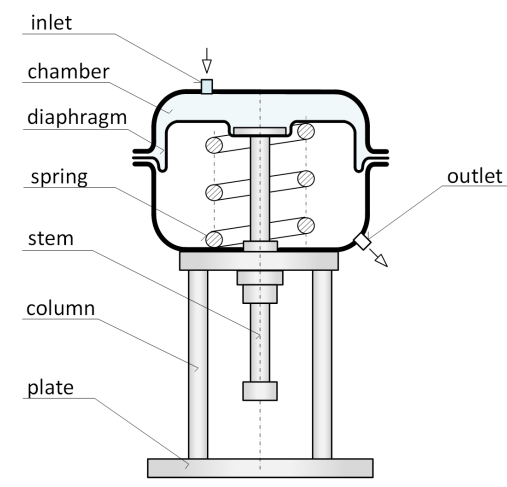

Figure 1. Single-action pneumatic actuator in normal mode.

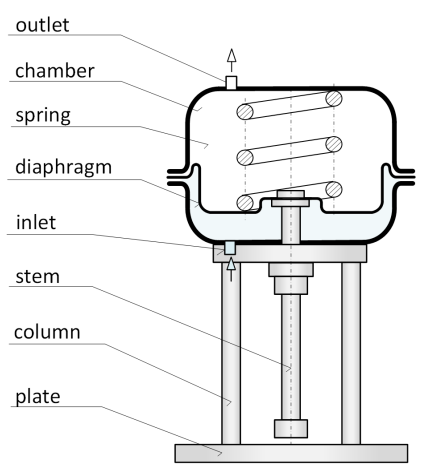

Figure 2. Single-action pneumatic actuator in reverse mode.

A single-action pneumatic actuator basically belongs to a normal- or reverse-action construction group. A spring or set of springs oppose the extension of the stem in normal-action mode (Fig. 1) or oppose the retraction of the stem for reverse-mode actuators (Fig. 2). The range of travel of the stem is adjusted from the construction and is limited based on the external and internal mechanical limiters, ie., determine the allowable travel of the control valve plug.

\section{Experiments}

An external electro-pneumatic transducer is commonly used for control of the air pressure entering the chamber of actuator. The position of the actuator stem is a nonlinear and ambiguous function of the air pressure in the chamber of the actuator. Fig. 3 shows the laboratory setup used for the investigation into the static and dynamic properties of an assembly consisting of a normal or reverse single-action pneumatic actuator and an electro-pneumatic transducer. The experiment setup applies a set of instruments that allow for measurements of the air pressure $\left(p_{s}\right)$ supply, air pressure $(p)$ in the chamber of the actuator, and the stem displacement $(x)$. A local real-time control and data acquisition system providing HART communication allows for conducting long-term static and short-term dynamic experiments controlled through the user application. Consider the interesting experiment results shown in Fig. 4, where a large step response of an assembly consisting of an electro-pneumatic transducer and a reverse action pneumatic actuator is demonstrated. 


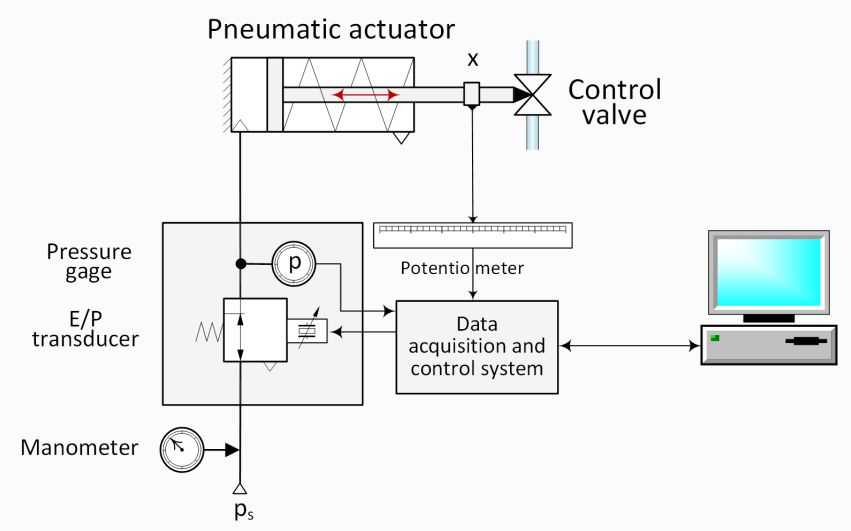

Figure 3. Schematic of laboratory setup for investigation into single-action pneumatic actuators.

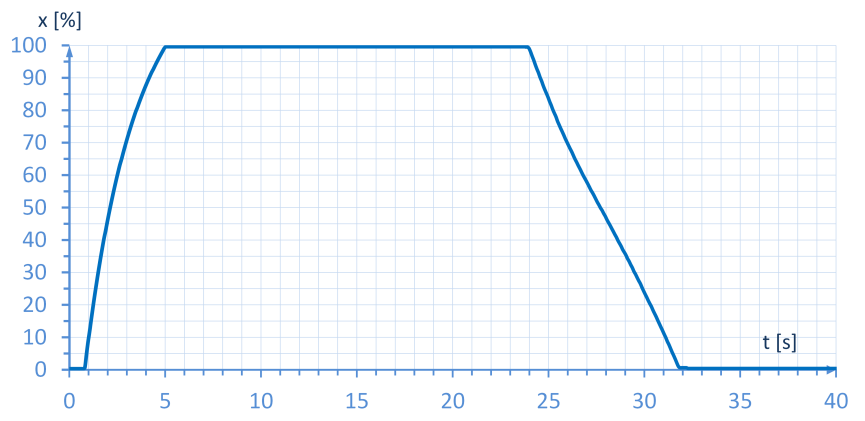

Figure 4. Large step response of type R1-400 reverse-acting actuator from Polna S.A.

From Fig. 4 it is easy to see that the slope of step response depends heavily on whether the stem extends or retracts. The evident asymmetry of the step response will be further referred to as the directionality of the dynamics of the actuator. The approximated effective transmittances of the stem extension $G_{1}(s)$ and retraction $G_{2}(s)$ can be described in the form of equation (1).

$$
G_{1}(s)=\frac{k_{1}}{T_{1} s+1} e^{-\tau_{1} s} ; \quad G_{2}(s)=\frac{k_{2}}{T_{2} s} e^{-\tau_{2} s}
$$

where $k_{1}$ are $k_{2}$ proportional gains, $T_{1}$ is a time constant of the first-order lag system, $T_{2}$ is a time constant of an integral system, and $\tau_{1}$ and $\tau_{2}$ are the transport delays.

Further, this study mainly focuses on describing the physics of the specific shape of the slopes of a step response. Therefore, the transport delays are not a concern.

The model of a pneumatic system consisting of double- and single-acting actuators and a servo-valve of an exoskeleton was presented in [8]. The modeling approach is based on the thermodynamic principles of energy and mass conservation. However, this study does not refer to nor discuss the effects of the asymmetry of the dynamics.

As the experiments conducted show, the effects of the asymmetry are not exceptional, and appear in both normal- and reverse-action actuators. Moreover, such effects occur for different types and executions of single-action actuators. It should be mentioned that the asymmetry of the dynamics is a factor to consider when developing a control system [9-12] or auto-tuner intended for positioners of the final electro-pneumatic control elements $[13,14]$. 
A systematic explanation of a phenomenon of asymmetry in the dynamics together with energy transformation issues is given based on the phenomenological (Sect. 4) and simulation (Sect. 6) models of the actuator. This can be considered as a main contribution of this paper based on theory, experiment and expertise.

To verify the developed models and the working hypotheses, a set of experiments and simulations that were conducted are reported in Sect. 7. The results achieved are summarized in Sect. 8.

\section{Preliminaries}

The following set of assumptions were adopted for modelling the dynamics of an assembly consisting of a single-action pneumatic actuator and electro-pneumatic transducer.

$1^{\circ}$ the dynamics of an electro-pneumatic transducer are negligible in comparison to those of a pneumatic actuator;

$2^{\circ}$ the viscous friction force is negligible;

$3^{\circ}$ the dry friction force of a pneumatic actuator is constant and non-negligible;

$4^{\circ}$ the air is compressible;

$5^{\circ}$ the hypothesis of an unchoked mass air flow is adopted;

$6^{\circ}$ the effective cross-sectional area of the diaphragm is constant;

$7^{\circ}$ the coefficient of elasticity of the diaphragm is negligible compared to that of the spring elasticity coefficient;

$8^{\circ}$ the absolute pressure in the unpressurized chamber of the actuator equals the external absolute pressure;

$9^{\circ}$ an isothermal gas transformation takes place in the actuator chamber;

$10^{\circ}$ none of the dynamic payload of the actuator stem is taken into account.

\section{Physical model}

Clearly, the modelling is an attempt to achieve an approximate description of the behavior of the system of interest. Typically, a set of simplifications and assumptions are adopted to develop a useful model. This is also the case in the present study because the aim is to describe the observed dynamic behavior of the single-action actuator, rather than the sophisticated physics behind it, as described in $[9,15,16]$.

First, the nonlinear dynamic model of an unlimited actuator stem traveling the distance $x$ is considered based on the equilibrium of the dynamic, kinetic, and static forces acting on the stem, as shown in formula (2)

$$
m_{l} \frac{d^{2} x}{d t^{2}}+\lambda \frac{d x}{d t}+k\left(x+x_{0}\right)+\left(k_{f}|x|+F\right) \cdot \operatorname{sgn}(x)=p \cdot A
$$

where $m_{l}$ is a lumped mass of moving elements, $x$ is the stem travel distance, $\lambda$ is the damping coefficient, $k$ is the spring constant, $x_{0}$ is the initial absolute position of the stem, $k_{f}$ is the viscous friction coefficient, $F$ is the Coulomb static dry friction force, $p$ is the overpressure in the actuator chamber, $A$ is the effective cross-sectional area of the diaphragm, and the initial conditions are as follows: $x=0, d x / d t=0$, $d^{2} x / d t^{2}=0$, and $p=0$.

In (2), the second-order damped oscillating system was adopted, in which the active force $(p \cdot A)$ is in equilibrium with the dynamic d'Alembert, damping, and friction forces. The friction force $f$ is modeled through a piecewise approximation of Striebeck's curve:

$$
f=\left(k_{f}|x|+F\right) \cdot \operatorname{sgn}(x)
$$

If necessary, this simple friction model may be replaced with the much more complex dynamic Bliman-Sorine or LuGre model [17], which captures a variety of phenomena associated with the friction 
effect, as applied in [18], where the adaptive LuGre model is used for the sliding mode control of a single action actuator of a turbocharger with a variable geometry. However, an estimation of the parameters of this model is a problem in itself.

The stem travel $x$ is bounded within the travel range limits. The theoretical unbounded stem travel equals:

$$
x_{a}=(p \cdot A-F) / k
$$

The air mass density of compressible air will be modeled based on the ideal gas equation:

$$
\rho=\frac{\delta}{R \cdot T} \cdot\left(p+p_{a}\right)
$$

where $R$ is a gas constant, $T$ is the absolute air temperature, $\delta$ is the air mol density, $p$ is the overpressure, and $p_{a}$ is the absolute ambient pressure at temperature $T$.

Hence, the total mass of the air collected in the chamber of the actuator is as follows:

$$
m=\left(V_{0}+x \cdot A\right) \cdot \rho=\left(V_{0}+x \cdot A\right) \cdot \frac{\delta}{R \cdot T} \cdot\left(p+p_{a}\right)
$$

where $A$ is the effective cross-sectional area of the diaphragm. Assuming $p_{a}=$ const, after a differentiation of (6), the following is obtained:

$$
\frac{d m}{d t}=\frac{\delta}{R \cdot T} \cdot\left[\left(V_{0}+x \cdot A\right) \frac{d p}{d t}+\left(p+p_{a}\right) \cdot A \cdot \frac{d x}{d t}\right]
$$

The unchoked mass flow of compressible air is adopted in this study. Therefore,

$$
\frac{d m}{d t}=C \cdot a \cdot \sqrt{2 \cdot \rho_{P_{1}} \cdot\left(P_{1}-P_{2}\right)}
$$

where $C$ is the dimensionless orifice flow coefficient, $a$ is the cross-sectional area of the orifice hole in an electro-pneumatic transducer, $\rho_{P_{1}}$ is the air mass density under absolute pressure $P_{1}, P_{1}$ is the upstream absolute air pressure, and $P_{2}$ is the downstream absolute air pressure.

\subsection{Model of leading edge of step response}

The velocity of the leading edge of the large step response shown in Fig. 4 seems to be continuously declining. We attempt to describe this phenomenon below. Substituting $\left(\rho_{P_{1}}=\frac{\delta}{R \cdot T} \cdot P_{1}\right)$ in (8) we obtain the following:

$$
\frac{d m}{d t}=C \cdot a \cdot \sqrt{2 \cdot \frac{\delta}{R \cdot T}} \cdot \sqrt{P_{1} \cdot\left(P_{1}-P_{2}\right)}
$$

By contrast,

$$
\frac{d m}{d t}=\frac{d V}{d t} \cdot \rho(p)=\frac{d V}{d t} \cdot \frac{\delta}{R \cdot T} \cdot P_{2}
$$

Substituting (9) with (10), and considering the following,

$$
\frac{d V}{d t}=A \cdot \frac{d x}{d t}
$$

we finally achieve

$$
\frac{d x}{d t}=C \cdot \frac{a}{A} \cdot \sqrt{2 \cdot \frac{R \cdot T}{\delta}} \cdot \sqrt{\frac{P_{1}}{P_{2}} \cdot\left(\frac{P_{1}}{P_{2}}-1\right)}
$$


For a sufficiently high $\left(P_{1} / P_{2}\right)$ ratio, i.e., for $P_{1} \gg P_{2}$,

$$
\frac{d x}{d t} \cong C \cdot \frac{a}{A} \cdot \sqrt{2 \cdot \frac{R \cdot T}{\delta}} \cdot \frac{P_{1}}{P_{2}}
$$

By applying the absolute supply pressure $\left(P_{1}=\right.$ const $)$, for $P_{1} \gg P_{2}$, we can conclude the following:

Conclusion 1: The velocity of the leading edge of the step response of the stem is approximately inversely proportional to the instantaneous value of the absolute air pressure in the chamber.

Fig. 5 shows this property in a unified form of (12), e.g., for $\left(\frac{1}{P_{1}} \cdot C \cdot \frac{a}{A} \cdot \sqrt{2 \cdot \frac{R \cdot T}{\delta}}=1\right)$.

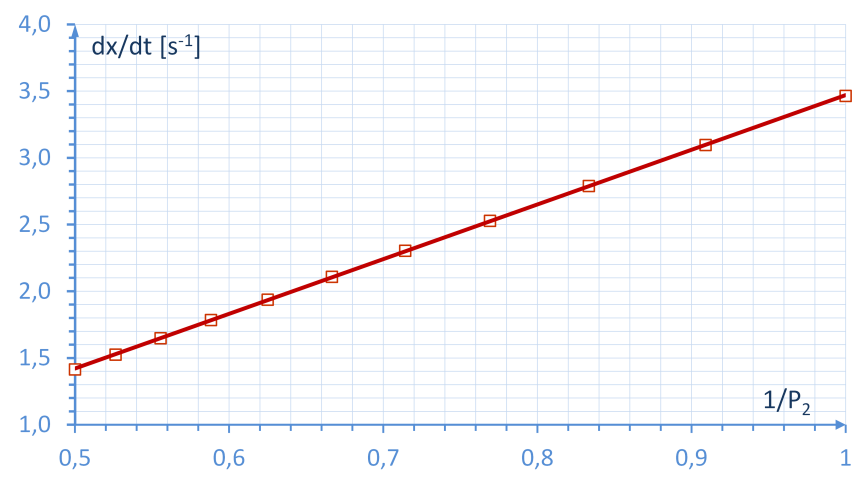

Figure 5. Unified theoretical velocity of the leading edge versus the reciprocal of $P_{2}$.

Eq. (12) implies some practical control strategy. To maintain a constant slope of the leading edge within the bounded range, the ratio $\left(P_{1} / P_{2}\right)$ should be kept constant. In this study, we assume that $\left(P_{1}=\right.$ const $)$, and we can therefore conclude the following.

Conclusion 2: There is no reason to expect a constant velocity of the leading edge of a large step response under a constant air supply pressure.

The upstream and downstream pressures during the chamber charging process are equal to $\left(P_{1}=\right.$ $\left.p_{s}+p_{a}\right)$ and $\left(P_{2}=p+p_{a}\right)$, respectively. Substituting $P_{1}$ and $P_{2}$ with (12), we obtain the following:

$$
\frac{d x}{d t}=C \cdot \frac{a}{A} \cdot \sqrt{2 \cdot \frac{R \cdot T}{\delta}} \cdot \sqrt{\frac{\left(p_{s}+p_{a}\right)}{\left(p+p_{a}\right)} \cdot \frac{\left(p_{s}-p\right)}{\left(p+p_{a}\right)}}
$$

Hence, the maximal stem velocity occurs under the minimal absolute pressure $P_{2}$, i.e., for $(p=0)$. In this case, we obtain from (14) an expression for the maximal velocity, i.e.,

$$
\left.\frac{d x}{d t}\right|_{\max }=C \cdot \frac{a}{A} \cdot \sqrt{2 \cdot \frac{R \cdot T}{\delta}} \cdot \sqrt{\frac{p_{s}}{p_{a}} \cdot\left(\frac{p_{s}}{p_{a}}+1\right)},
$$

and for $\left(p_{s} \gg p_{a}\right)$ and $\left(p_{a}=\right.$ const $)$, we have the following:

$$
\left.\frac{d x}{d t}\right|_{\max } \cong C \cdot \frac{a}{A} \cdot \sqrt{2 \cdot \frac{R \cdot T}{\delta}} \cdot \frac{p_{s}}{p_{a}} .
$$

Conclusion 3: The maximal velocity of the leading edge of the step response is approximately proportional to the supply pressure. Hence, the ratio of the maximal velocity of the leading edge to the supply pressure is approximately constant. 
When charging, the chamber pressure tends to increase, and the velocity of the stem decreases. According to (14), the minimal velocity is as follows:

$$
\left.\frac{d x}{d t}\right|_{\text {min }}=\lim _{p \rightarrow p_{s}} C \cdot \frac{a}{A} \cdot \sqrt{2 \cdot \frac{R \cdot T}{\delta}} \cdot \sqrt{\frac{\left(p_{s}+p_{a}\right)}{\left(p+p_{a}\right)} \cdot \frac{\left(p_{s}-p\right)}{\left(p+p_{a}\right)}}=0
$$

Conclusion 4: The minimal velocity of the leading edge of the step response tends to be zero.

Conclusions $1-4$ are in accordance with the experiment observations.

\subsection{Model of the falling edge of the step response}

The velocity of the falling edge of the large step response shown in Fig. 4 appears to be approximately constant. Below, we attempt to explain this phenomenon. Clearly, a chamber discharge follows a general equation (9). However, in this case, the air mass flow is proportional to the air density under pressure $P_{1}$ instead of $P_{2}$, as during charging process. Therefore,

$$
\frac{d m}{d t}=\frac{d V}{d t} \cdot \rho(p)=\frac{d V}{d t} \cdot \frac{\delta}{R \cdot T} \cdot P_{1}
$$

Substituting (18) with (9) and considering (11), we finally obtain the following:

$$
\frac{d x}{d t}=C \cdot \frac{a}{A} \cdot \sqrt{2 \cdot \frac{R \cdot T}{\delta}} \cdot \sqrt{1-\frac{P_{2}}{P_{1}}}
$$

Therefore, for a sufficiently low $\left(P_{2} / P 1\right)$, i.e., for $\left(P_{2} \ll P_{1}\right)$, we have the following:

$$
\frac{d x}{d t} \cong C \cdot \frac{a}{A} \cdot \sqrt{2 \cdot \frac{R \cdot T}{\delta}}
$$

Conclusion 5: The stem velocity of the falling edge of the stem response is approximately constant for a sufficiently low $\left(P_{2} / P_{1}\right)$ ratio.

This conclusion is consistent with the experiment observations, which confirms the assumptions adopted in Sect. 2 to a certain extent.

Conclusion (5) has also a practical meaning. Assume that the pressure bench set range equals $\left(p_{b l} . . p_{b h}\right)$. According to (19), the utmost velocities within the pressure bench range are equal to the following:

$$
\begin{aligned}
& \left.\frac{d x}{d t}\right|_{l}=C \cdot \frac{a}{A} \cdot \sqrt{2 \cdot \frac{R \cdot T}{\delta}} \cdot \sqrt{\frac{p_{b l}}{p_{b l}+p_{a}}} \\
& \left.\frac{d x}{d t}\right|_{h}=C \cdot \frac{a}{A} \cdot \sqrt{2 \cdot \frac{R \cdot T}{\delta}} \cdot \sqrt{\frac{p_{b h}}{p_{b h}+p_{a}}}
\end{aligned}
$$

Therefore, the relative change in velocities within the pressure bench set is equal to

$$
\frac{\left.\frac{d x}{d t}\right|_{h}-\left.\frac{d x}{d t}\right|_{l}}{\left.\frac{d x}{d t}\right|_{h}}=1-\sqrt{\frac{p_{b l}}{p_{b h}} \cdot \frac{p_{b h}+p_{a}}{p_{b l}+p_{a}}}=1-\sqrt{\frac{1+\frac{p_{a}}{p_{b h}}}{1+\frac{p_{a}}{p_{b l}}}}
$$

From (23), an obvious conclusion can be drawn in which, if the limit values $p_{b l}$ and $p_{b h}$ of the pressure bench set are close, then the change in velocity is small. Much more important, however, is the following statement. 
Conclusion 6: The change in relative stem velocity within an arbitrary fixed pressure range does not depend on the constructive parameters of the actuator and/or electro-pneumatic transducer, or the supply pressure.

For $\left(p_{b l} \gg p_{a}\right)$, the relative change in velocity is close to zero for the following reason:

$$
\lim _{\frac{p_{a}}{p_{b l}} \rightarrow 0}\left(1-\sqrt{\frac{1+\frac{p_{a}}{p_{b h}}}{1+\frac{p_{a}}{p_{b l}}}}\right)=0
$$

In this case, the slope of the falling edge of the step response is almost constant within the set range of the pressure bench.

Example 1: Consider the reverse acting multi-spring actuator type R1-400 from Polna S.A. [5]. This actuator may be equipped with different numbers of springs, as shown in Tab. 1. The step response of this actuator equipped with six springs is depicted in Fig. 4. Let the absolute ambient pressure equal $p_{a}=100 \mathrm{kPa}$. The results of the relative change in velocity within different pressure bench sets are shown in Tab. 1.

Table 1. Relative change in trailing edge slope versus pressure bench set for reverse-acting multi-spring actuator type R1-400 from Polna S.A.

\begin{tabular}{ccccc}
\hline $\begin{array}{c}\text { No. of } \\
\text { springs }\end{array}$ & $\begin{array}{c}\mathbf{p}_{\mathrm{bl}} \\
{[\mathbf{k P a}]}\end{array}$ & $\begin{array}{c}\mathbf{p}_{\mathrm{bh}} \\
{[\mathbf{k P a}]}\end{array}$ & $\begin{array}{c}\mathbf{p}_{\mathrm{bh}} \mathbf{p}_{\mathrm{bl}} \\
{[\mathbf{k P a}]}\end{array}$ & $\begin{array}{c}\text { Relative change } \\
\text { of velocity (Eq. 23) }\end{array}$ \\
\hline 3 & 20 & 100 & 80 & -0.290 \\
6 & 80 & 240 & 160 & -0.200 \\
12 & 180 & 380 & 200 & -0.175 \\
\hline
\end{tabular}

By discharging the chamber, the upstream and downstream pressures are equal to $\left(P_{1}=p+p_{a}\right)$ and $\left(P_{2}=p_{a}\right)$, respectively. Substituting $P_{1}$ and $P_{2}$ with (19), we obtain the following:

$$
\frac{d x}{d t}=C \cdot \frac{a}{A} \cdot \sqrt{2 \cdot \frac{R \cdot T}{\delta}} \cdot \sqrt{\frac{\frac{p}{p_{a}}}{1+\frac{p}{p_{a}}}}
$$

Hence, the higher the chamber pressure is, the higher the velocity of the falling edge. For a sufficiently high ratio $\left(p / p_{a}\right)$,

$$
\frac{d x}{d t} \cong C \cdot \frac{a}{A} \cdot \sqrt{2 \cdot \frac{R \cdot T}{\delta}}=\text { const }
$$

Conclusion 7: The stem velocity is roughly constant through the discharge of the actuator chamber.

This clarifies the shape of the falling edge of the step response shown in Fig. 4. Fig. 6 indicates the normalized $\left(C \cdot \frac{a}{A} \cdot \sqrt{2 \cdot \frac{R \cdot T}{\delta}}=1\right)$ falling edge velocity versus the relative chamber pressure. It can be seen that, for $\left(p>5 p_{a}\right)$, the velocity of the falling edge of the step response is fairly constant. The yellow area shows that the pressure range $(180 . .380 \mathrm{kPa})$ is equal to the pressure bench set, as in Example 1 . As can be seen from Fig. 6, the normalized change in the falling slope within this range is no greater than $10 \%$. 


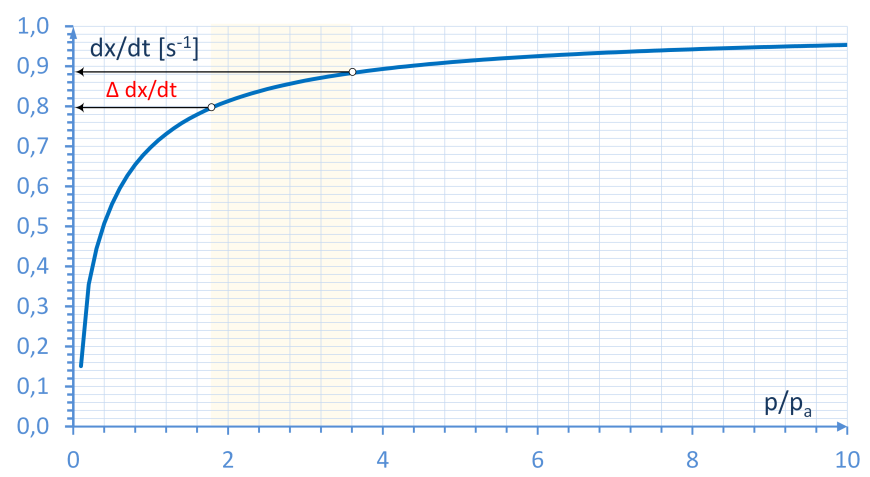

Figure 6. Normalized theoretical velocity of the stem of the actuator by the chamber discharge versus the $p / p_{a}$ ratio.

From (25), the maximal stem velocity occurs through the maximal chamber pressure, i.e., for $\left(p=p_{s}\right)$,

$$
\left.\frac{d x}{d t}\right|_{\max }=C \cdot \frac{a}{A} \cdot \sqrt{2 \cdot \frac{R \cdot T}{\delta}} \cdot \sqrt{\frac{\frac{p_{s}}{p_{a}}}{1+\frac{p_{s}}{p_{a}}}},
$$

and for $\left(p_{s} \gg p_{a}\right)$ and $\left(p_{a}=\right.$ const $)$,

$$
\left.\frac{d x}{d t}\right|_{\max } \cong C \cdot \frac{a}{A} \cdot \sqrt{2 \cdot \frac{R \cdot T}{\delta}}
$$

Conclusion 8: The maximal slope of the falling edge of the step response depends mainly on the constructive parameters of the actuator and electro-pneumatic transducer.

During the discharging process, the air pressure in the chamber tends to reach the ambient pressure, and the velocity of the stem decreases. From (25), we obtain the minimal velocity:

$$
\left.\frac{d x}{d t}\right|_{\min }=\lim _{p \rightarrow p_{s}} C \cdot \frac{a}{A} \cdot \sqrt{2 \cdot \frac{R \cdot T}{\delta}} \cdot \sqrt{0.5} \neq 0
$$

Conclusion 9: The theoretical minimal velocity of the falling edge of the step response is a value other than zero.

Comparing (28) and (29), we can formulate the following simple and practical rule.

Conclusion 10: The theoretical ratio of the maximal to minimal velocity of the falling edge of the step response is no greater than $\sqrt{2}$.

In other words, the relative theoretical change in velocity of the falling edge of a large step response does not exceed $29.3 \%$.

This rule can be useful in auto-tuning algorithms intended for positioners of the final control elements with single-action pneumatic actuators for an estimation of the minimal ramp generator time constant guaranteeing a symmetrical response of the final control element to the stepwise setpoint signal. Moreover, we have the following:

Conclusion 11: The relative theoretical change in velocity of the falling edge of the step response does not depend on the actuator and/or electro-pneumatic transducer parameters.

In the practice, however, the real stem travel covers only a portion of its theoretical travel range, and the relative change in velocity is much less than $29.3 \%$. This gives the assumption that the relative change 
in velocity is roughly negligible. This statement explains well the shape of the falling edge of the step response observed in Fig. 4.

\subsection{Relation between velocities of leading and trailing edges}

In this subsection, we describe the relation between the leading and trailing slopes of a large step response. First, let us assume that the maximum velocity of the leading edge is greater than the minimum velocity of the trailing edge. Comparing (15) and (29), we obtain the following:

$$
\frac{p_{s}}{p_{a}} \cdot\left(\frac{p_{s}}{p_{a}}+1\right) \geq \sqrt{0.5}
$$

From (30), the maximum velocity of the leading edge is greater than the maximum velocity of the trailing edge for $p_{s} / p_{a} \geq 0.478$. In practice, $p_{s} / p_{a} \geq 1.0$, and thus the following can be derived:

Conclusion 12: In a real system, the maximum velocity of the leading edge of a large step response is greater than the minimum velocity of the trailing edge.

Simultaneously, from (17) and (27), the following can be inferred:

Conclusion 13: The minimum velocity of the leading edge of a large step response is lower than the maximum velocity of the trailing edge.

Therefore, from conclusions (12) and (13), we can infer that there are existing conditions for which the velocities in both directions are equal. For such an inference, we will next compare (14) with (25). Let us first determine the necessary conditions for which the velocity of the leading slope is greater than or equal to that of the trailing slope, i.e.,

$$
\frac{\left(p_{s}+p_{a}\right)}{\left(p+p_{a}\right)} \cdot \frac{\left(p_{s}-p\right)}{\left(p+p_{a}\right)} \geq \frac{p}{\left(p+p_{a}\right)}
$$

From (31), it follows that

$$
\frac{\left(p_{s}+p_{a}\right)}{\left(p+p_{a}\right)} \cdot \frac{\left(p_{s}-p\right)}{p} \geq 1
$$

After a transformation of the inequality (32), we obtain the following:

$$
\left(\frac{p}{p_{s}}\right)^{2}+\left(2 \cdot \frac{p_{a}}{p_{s}}+1\right)\left(\frac{p}{p_{s}}\right)-\left(\frac{p_{a}}{p_{s}}+1\right) \leq 0
$$

This quadratic inequality is satisfied for the following:

$$
\left[-\left(\frac{p_{a}}{p_{s}}+\frac{1}{2}\right)-\frac{1}{2} \sqrt{\Delta}\right] \leq \frac{p}{p_{s}} \leq\left[-\left(\frac{p_{a}}{p_{s}}+\frac{1}{2}\right)+\frac{1}{2} \sqrt{\Delta}\right],
$$

where $\Delta=\left(2 p_{a} / p_{s}+1\right)^{2}+4\left(p_{a} / p_{s}+1\right)$. Taking into consideration that, with $\left(p / p_{s} \geq 0\right)$, the inequality (34) is simplified as follows:

$$
0 \leq \frac{p}{p_{s}} \leq\left[-\left(\frac{p_{a}}{p_{s}}+\frac{1}{2}\right)+\frac{1}{2} \sqrt{\Delta}\right]
$$

Clearly, the right boundary of the inequality (35) cannot be negative. Therefore,

$$
-\left(\frac{p_{a}}{p_{s}}+\frac{1}{2}\right)+\frac{1}{2} \sqrt{\Delta} \geq 0
$$


It is easy to show that the inequality (36) is almost always satisfied for $\left(p_{a} / p_{s} \geq-1\right)$. By contrast, $p \leq p_{s}$, and therefore we have the following:

$$
-\left(\frac{p_{a}}{p_{s}}+\frac{1}{2}\right)+\frac{1}{2} \sqrt{\Delta} \leq 1
$$

This inequality is satisfied for $\left(p_{a} / p_{s}\right) \geq-4 / 7$, i.e., it is always satisfied. The zero crossing points of inequality (35) are shown in Fig. 7.

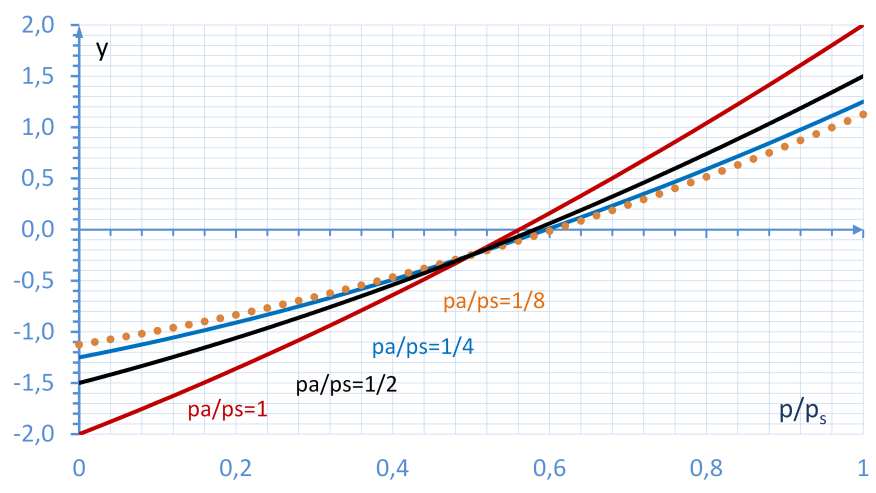

Figure 7. Illustration of solution of inequality (35).

The curve shown in Fig. 8 is much more informative, demonstrating the normalized air pressure in the chamber versus the relative actuator supply pressure. For convenience, the range of typical supply pressures of industrial actuators $(140 . .800 \mathrm{kPa})$ is shown in the light-red area. As can be seen, the normalized air pressure in the chamber for which the inequality (35) is satisfied is almost constant compared to the relative supply pressure.

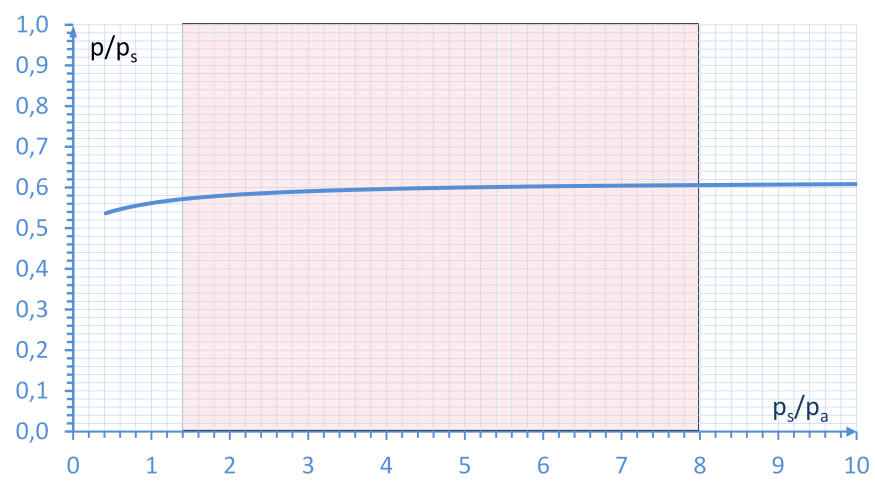

Figure 8. Normalized critical air pressure in the chamber versus relative actuator supply pressure.

Now, we fix the asymptote of the normalized critical air pressure in the chamber. From (35), we obtain the following:

$$
\lim _{\frac{p_{s}}{p_{a}} \rightarrow \infty} \frac{p}{p_{s}}=-\left(\frac{1}{\frac{p_{s}}{p_{a}}}+\frac{1}{2}\right)+\frac{1}{2} \sqrt{\left(2 \frac{1}{\frac{p_{s}}{p_{a}}}+1\right)^{2}+4\left(\frac{1}{\frac{p_{s}}{p_{a}}}+1\right)}=0.618
$$

Conclusion 14: The velocity of the trailing edge is greater than the velocity of the leading edge when the air pressure in the chamber surpasses the 0.618 value of the supply pressure. 
Example 2: For the actuator, let the nominal supply pressure $p_{s}=400 \mathrm{kPa}$ and ambient pressure $p_{a}=100 \mathrm{kPa}$, as shown in Example 1. The critical ratio of $\left(p / p_{s}\right)$ calculated from (35) equals $\left(p / p_{s}\right)=-\left(p_{a} / p_{s}+1 / 2\right)+$ $1 / 2 \sqrt{\Delta}=-(1 / 4+1 / 2)+1 / 2 \sqrt{(2 \cdot 1 / 4+1)^{2}+4(1 / 4+1)}=0.596$. Therefore, for $p \geq 0,596 * 400 \mathrm{kPa}=$ $238 \mathrm{kPa}$, we can expect a greater velocity of the trailing edge than the leading one. If we choose the actuator with the pressure bench set $[20 . .100 \mathrm{kPa}]$, then for any pressure within this range, the velocity of the leading edge will be greater than that of the trailing edge. The same observation holds for a pressure bench range of $[80 . .240 \mathrm{kPa}]$. However, if we consider applying a pressure bench range of $[180 . .380 \mathrm{kPa}]$ rather than a pressure below $238 \mathrm{kPa}$, the velocity of the trailing edge will be lower, whereas for pressures greater than $238 \mathrm{kPa}$, it will be greater.

Conclusion 15: By appropriately choosing the pressure bench set, it is possible to "symmetrize" the slopes of the step response of a single action-actuator.

Conclusion (15) is important for an identification of the parameters for the controller of the stem position.

Conclusion 16: When searching for the maximal stem velocity, the best choice is to determine the instantaneous value of the velocity just after the stem starts to move.

\section{Effectiveness of energy conversion}

The energy of outflowing air is less than the energy of inflowing air because of energy losses during the charging-discharging cycle. When charging the chamber, the air supply pressure is constant, and the pressure in the chamber grows. However, when the chamber is discharged, the pressure decreases and the outflow pressure is almost constant. Therefore, the following holds:

$$
\int_{0}^{\infty} p_{s} \cdot q_{i}(t) d t>\int_{0}^{\infty} p \cdot q_{o}(t) d t
$$

where $q_{i}$ is the air inflow rate, and $q_{o}$ is the air outflow rate.

Substituting $q_{i}(t)=A\left(d x_{i}\right) / d t$ and $q_{o}(t)=A\left(d x_{o}\right) / d t$ in (39), we obtain the following:

$$
A \int_{0}^{\infty} p_{s} \cdot q_{i}(t) \cdot \frac{d x_{i}}{d t} d t>A \int_{0}^{\infty} p \cdot q_{o}(t) \cdot \frac{d x_{0}}{d t} d t
$$

whereas for $p_{s} \geq p$ and $d x_{o}=A / k \cdot d p$

$$
A \int_{0}^{x_{n}} p_{s} d x_{i}>\frac{A^{2}}{k} \int_{0}^{p_{s}} p d p
$$

we have

$$
A \cdot p_{s} \cdot x_{n}>\frac{1}{2} \cdot A \cdot\left(\frac{A}{k} p_{s}\right) \cdot p_{s} .
$$

The left side of the inequality (42) expresses the energy of the air that is delivered to move the stem within the nominal travel range. The right side of the inequality (42) expresses the energy of the air leaving the chamber. Substituting $\left(x_{n} \cong \frac{A}{k} p_{s}\right)$ and $\left(E_{i}=A \cdot p_{s} \cdot x_{n}\right)$ in (42), we obtain the following:

$$
E_{o}=\frac{1}{2} E_{i}
$$

Conclusion 17: The maximal theoretical energy efficiency of the charging-discharging cycle in a single action pneumatic actuator equals $50 \%$. 
The total energy required to move the stem within the travel range and completely pressurize the chamber of actuator delivered during the isothermal process is equal to the following:

$$
E_{i}=E_{s_{0}}+E_{s}+E_{a}+E_{F}^{\prime}+E_{f}^{\prime}+E_{w}^{\prime}
$$

where $E_{s_{0}}$ is the initial potential energy accumulated in the pre-compressed spring, $E_{s}$ is the potential energy accumulated in the spring, $E_{a}$ is the potential energy accumulated in the compressed air in the chamber, $E_{F}^{\prime}$ is the energy dissipated by dry friction, $E_{f}^{\prime}$ is the energy dissipated by viscous friction, and $E_{w}^{\prime}$ is the amount of energy delivered to the load.

However, the energy accumulated in the chamber will apply the useful work $E_{w}^{\prime \prime}$ during discharging process. Therefore, we have the following:

$$
E_{s_{0}}+E_{s}+E_{a}=E_{F}^{\prime \prime}+E_{f}^{\prime \prime}+E_{w}^{\prime \prime}+E_{o}
$$

where $E_{F}^{\prime \prime}$ is the energy dissipated by dry friction, and $E_{f}^{\prime \prime}$ is the energy dissipated by viscous friction.

Substituting (45) with (44), we obtain the following:

$$
E_{i}=\left(E_{w}^{\prime}+E_{w}^{\prime \prime}\right)+\left(E_{F}^{\prime}+E_{f}^{\prime}+E_{F}^{\prime \prime}+E_{f}^{\prime \prime}\right)
$$

From (46), the energy provided to the actuator is used for the useful work and losses. Hence, the energy effectiveness equals

$$
\frac{E_{w}^{\prime}+E_{w}^{\prime \prime}}{E_{i}}=\left(1-\frac{E_{o}}{E_{i}}\right)-\frac{1}{E_{i}}\left(E_{F}^{\prime}+E_{f}^{\prime}+E_{F}^{\prime \prime}+E_{f}^{\prime \prime}\right) .
$$

Regarding (43) and substituting $E_{w}=\left(E_{w}^{\prime}+E_{w}^{\prime \prime}\right) ; E_{F}=\left(E_{F}^{\prime}+E_{f}^{\prime}+E_{F}^{\prime \prime}+E_{f}^{\prime \prime}\right)$, we obtain the following:

$$
\frac{E_{w}}{E_{i}}=\frac{1}{2}-\frac{E_{F}}{E_{i}} .
$$

In addition, $\left(E_{F}>0\right)$

$$
\frac{E_{w}}{E_{i}}<\frac{1}{2}
$$

This allows us to refine conclusion (17).

Conclusion 18: The energy efficiency of a single action pneumatic actuator is less than $50 \%$ based on the ratio of the total energy losses related to the energy of the air pressure entering the actuator.

\section{Simulation model}

Simulations of a single-action pneumatic actuator were conducted in a MATLAB-Simulink environment. Basically, the simulation model consists of two inter-coupled submodels, namely, a submodel of the dynamics of the pneumatic actuator and a submodel of the air pressure in the actuator chamber. A general schematic of the model structure is depicted in Fig. 9. 


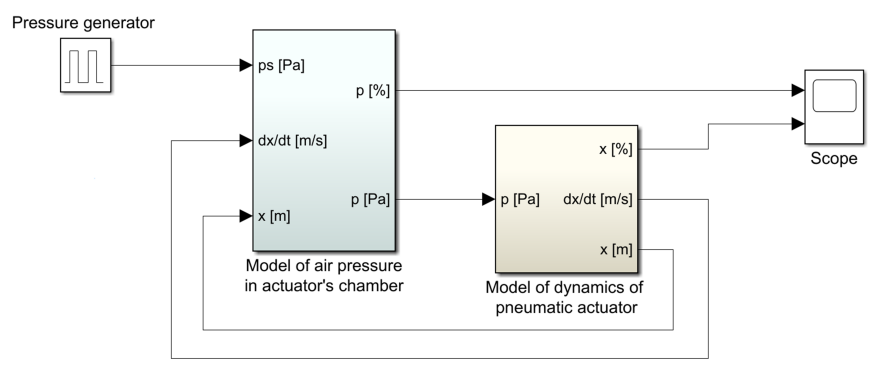

Figure 9. General structure of the model of a single-action spring-and-diaphragm pneumatic actuator in a MATLAB-Simulink environment.

The model was developed based on the analytical equations (2), (7) and (8) introduced in Sect. 4. These equations refer to the physical laws, and therefore the model considered should be recognized as a general model. However, for verification of the goals, the parameters of the model were fixed and tuned to mimic the two actuators, namely, a reverse-acting type R1-400 from Polna S.A. and a normal-acting type 37-13 from Masoneilan. The set of parameters used for simulation purposes are given in Tab. A1 in the appendix. This set contains a subset of known and unknown parameters that were adjusted during the simulation process.

\subsection{Submodel of dynamics of pneumatic actuator}

The structure of the submodel used for the dynamics of the pneumatic actuator is shown in Fig. 10.

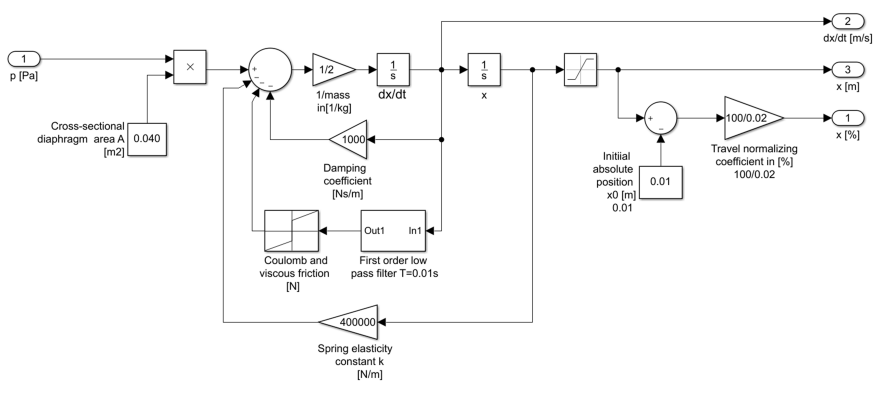

Figure 10. The structure of the submodel used for the dynamics of a single-action spring-and-diaphragm pneumatic actuator in a MATLAB-Simulink environment.

This reflects the dynamics of the actuator given in (2) within the physical bounds defined by the travel range and the pressure bench set. The travel range is the portion of theoretical stem travel bounded by the external mechanical limiters. The pressure bench set $p_{b n}$ defines the nominal range of air pressure necessary to move the stem within the nominal travel range. Clearly, the real travel range and the pressure bench set may differ from the nominal values. The static characteristics of the pneumatic actuator obtained from this model is shown in Fig. 11. The dynamic model is exploited further in Sect. 7. 


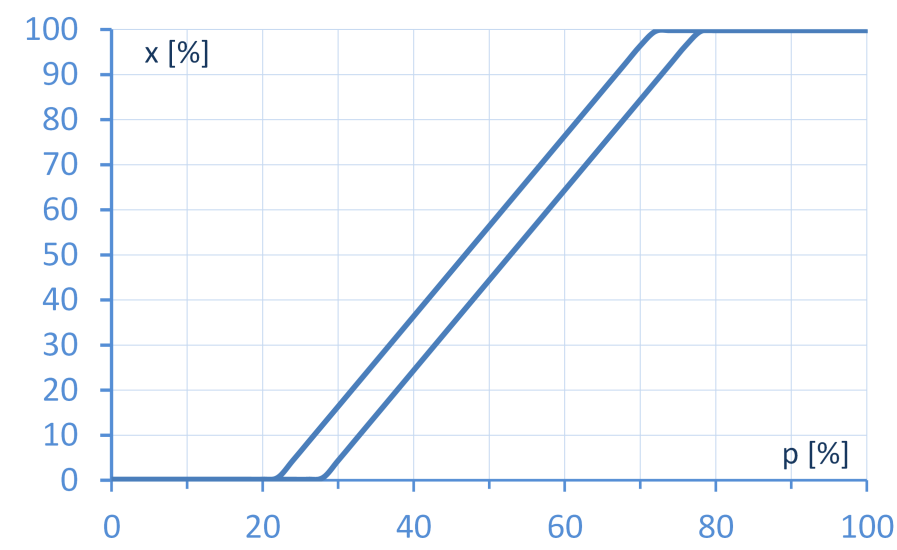

Figure 11. Simulation of static characteristics of reverse-acting actuator R1-400 from Polna S.A.

\subsection{Submodel of air pressure}

A submodel of the air pressure build-up process is shown in Fig. 12. This reflects the dynamics of the pressure development process in accordance with the analytical description of the physics, under the assumptions considered in Sections 2 and 4 . The dynamics of the entire actuating assembly is principally determined based on the dynamics of the pressure build-up process. Therefore, it is feasible to recognize the properties of this part of the actuator model to clarify the asymmetry. As shown in Eqs (7) and (8), these properties result from the dynamics of the air mass inflow and outflow into or out of the chamber of the actuator.

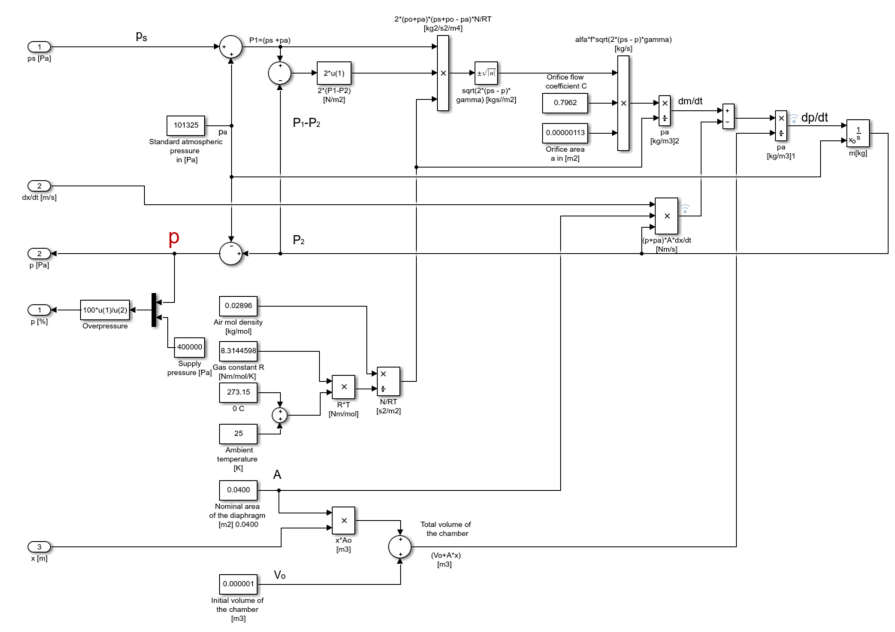

Figure 12. Structure of the air-pressure submodel of a single-action spring-and-diaphragm pneumatic actuator in the MATLAB-Simulink environment.

The submodel of the air-pressure build-up process was tuned and verified. The admissibility of assumptions adopted in Sect. 2 have been proven indirectly through conformity tests conducted in simulations and from experiment data taken from real actuators. The overlapping data between the experiment and the simulation results are shown in Fig. 13 for a reverse-action actuator R-400 from Polna S.A., and in Fig. 14 for a normal-action actuator 37-13 from Masoneilan. The matching of the models with the experiment data indicate the satisfactory nature of the evaluation. 


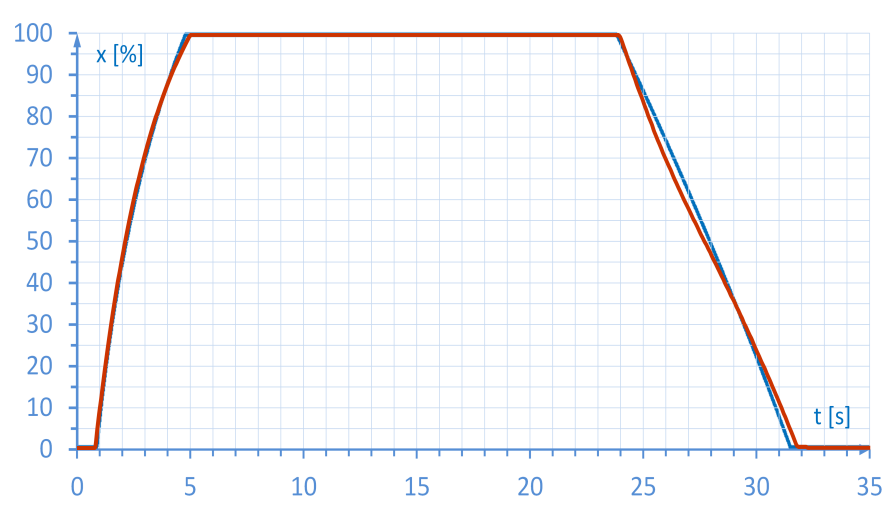

Figure 13. Step response of investigated (red line) and simulated (dark blue line) reverse- and single-action pneumatic actuator, type R1-400, from Polna S.A.

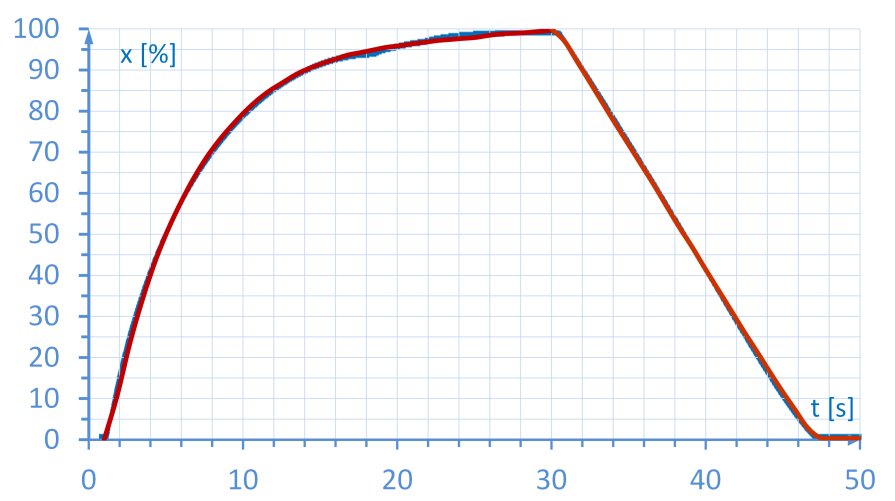

Figure 14. Step response of investigated (red line) and simulated (dark blue line) normal-action pneumatic actuator, type 37-13, from Masoneilan. The upper position of the mechanical limiter is dismounted.

\section{Discussion}

In principle, the theoretical deliberations described in Sect. 4, univocally explain the shape of the large step response of the actuator. As stated in conclusion (1), the leading slope is approximately inversely proportional to the instantaneous value of the absolute air pressure in the chamber, whereas according to conclusion (7), the trailing slope is roughly constant. The open problem is now an explanation of the root cause of this phenomenon. In this section, we discuss six working hypotheses, all of which were verified through testing conducted using the simulation model of actuator R1-400 presented in Sect. 6.

Hypothesis 1: The asymmetry is caused by the directional action of the springs on the actuator's diaphragm.

The spring or springs oppose the stem travel when they are compressed, and thus slow down the stem movement, or by contrast, act in the direction of the stem movement when the springs are relaxed.

Hypothesis 1 was not confirmed. If true, the asymmetry should disappear if the spring forces tend toward zero. This can be simulated by introducing variations into the spring constant. The results are illustrated in Fig. 15, where the spring constant was artificially differentiated in both directions of the stem movement. An asymmetry of the shape response still exists. The change in spring constant influences the time constants of the effective transmittances (1), but not the asymmetry of the response.

Hypothesis 2: The asymmetry is caused by a non-zero initial spring compression force.

Hypothesis 2 was also not confirmed. If true, the asymmetry should disappear for an initial spring compression force close to zero. This is not the case, however. The change in the initial spring compression force influences the time constants of the effective transmittances (1), but not the asymmetry of the step response. The influence of the initial spring compression force on the time constants is illustrated in Fig. 16, 


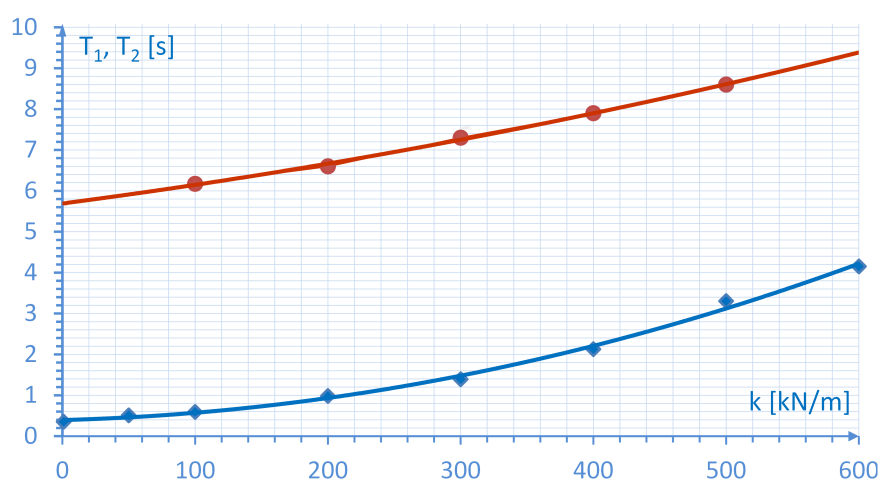

Figure 15. Influence of spring constant $k$ on the effective time constant: $T_{1}$ (dark blue line) and $T_{2}$ (red line).

where the initial compression force $F_{0}$ of the spring is changed within the range of $0 . .4000 N$, i.e., at up to $50 \%$ of the nominal active force.

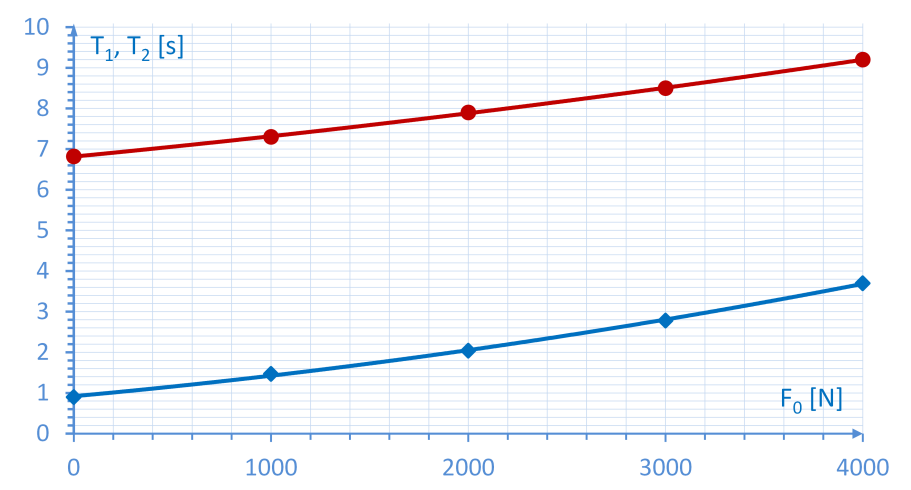

Figure 16. Influence of initial spring compression on effective time constant: $T_{1}$ (dark blue line) and $T_{2}$ (red line).

Hypothesis 3: The asymmetry is caused by the application of the mechanical limiters of the stem travel. Therefore, only a portion of the nominal stem travel is observed in response to a large step excitation.

Hypothesis 3 was clearly not confirmed. The asymmetry of the dynamics does not depend on the settings of the mechanical limiters of the stem travel. The dynamics of the pressure build-up or discharge process is unaffected. The change in travel range exclusively influences the time constants of the effective time transmittances, as shown in Fig. 17, where the stem travel range is changed to within $25 . .100 \%$ of the nominal travel range.

Hypothesis 4: The asymmetry results from the pressure bench set.

The pressure bench set is a range of air pressures that should be applied to move the stem of the actuator within the requested travel range.

Hypothesis 4 was also not confirmed. The asymmetry does not result from the pressure bench set. The pressure bench set, together with the travel range, indirectly defines the spring constant. However, for the same spring, the asymmetry does not depend on the travel range, as discussed in Hypothesis 3, nor the spring constant, as shown in the discussion of Hypothesis 1. Therefore, we can infer that the asymmetry is not caused by a pressure bench set. Clearly, the choice of pressure bench range influences the effective time constants shown in Fig. 18.

Hypothesis 5: The asymmetry is caused by the asymmetry in the outflow and inflow pneumatic resistance of an electro-pneumatic transducer driving the actuator chamber. 


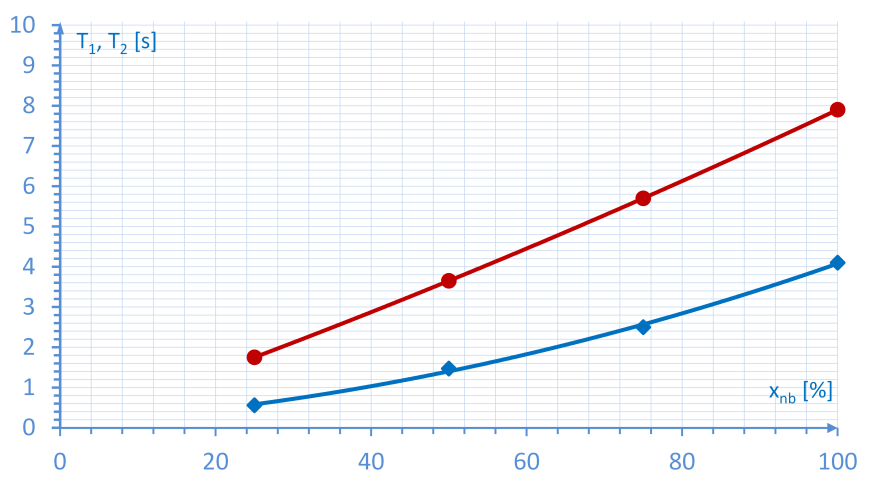

Figure 17. Influence of stem travel range on effective time constant: $T_{1}$ (dark blue line) and $T_{2}$ (red line).

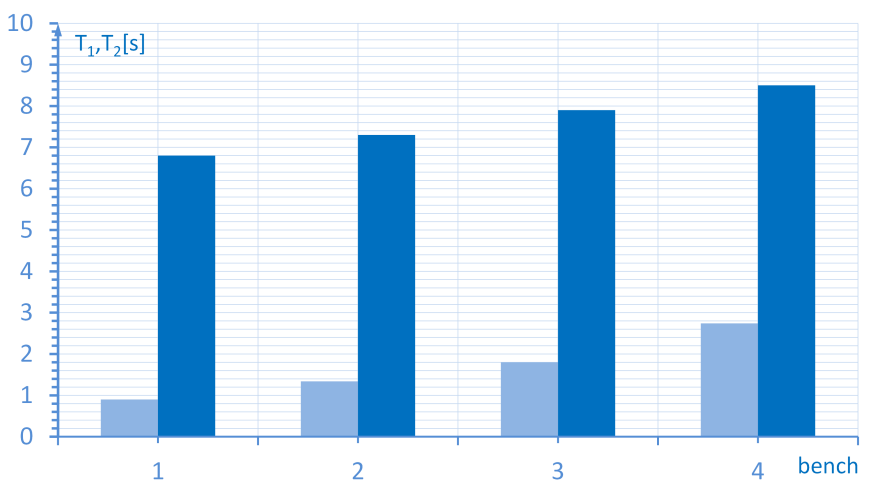

Figure 18. Influence of pressure bench set on effective time constant: $T_{1}$ (light blue bars) and $T_{2}$ (dark blue bar). The bench set classes are as follows: 1) $(80 . .240 \mathrm{kPa}), 2)(120 . .280 \mathrm{kPa}), 3)(160 . .320 \mathrm{kPa})$, and 4$)(200-360$ $\mathrm{kPa})$.

Hypothesis 5 was not confirmed. The asymmetry of the outflow and inflow pneumatic resistance of the electro-pneumatic transducer does not influence the asymmetry of the dynamics. However, it does influence the parameters of the effective transmittance (1). The influence of the pneumatic resistance on the effective time constants, $T_{1}$ and $T_{2}$, is illustrated in Fig. 19.

Hypothesis 6: The asymmetry is an effect of the different conditions of the gas transformation depending on whether the chamber is charged or discharged.

Hypothesis 6 is confirmed. The asymmetry of the dynamics is a result of the different thermodynamic air processes in both the stem travel directions. The air mass inflow diminishes with the increase in pressure in the chamber. Simultaneously, the volume of the chamber increases and the pressure build-up process successively slows, decreasing the velocity of the stem, which is approximately proportional to the change in air pressure in the chamber, as is typically found during the self-saturation process.

During the air outflow, the situation is significantly different. On the one hand, the air outflow diminishes the pressure in the chamber, whereas on the other hand, the process of the shrinking volume of the chamber slows the pressure decrease. Therefore, the deceleration of stem movement will also slow, which is characteristic for an astatic-like system.

\section{Summary}

This paper discussed the effect of the asymmetry of a large step response observable in the family of electromechanical single-action fluid power devices. The aim of this study was to describe this phenomenon and draw both theoretical and practical conclusions. 


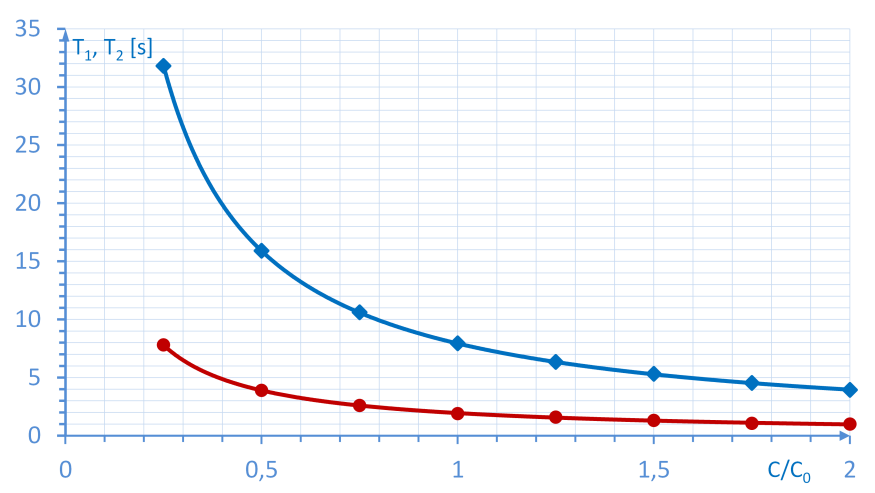

Figure 19. The influence of the pneumatic resistance on the effective time constants, $T_{1}$ (dark blue line) and $T_{2}$ (red line), versus the relative flow coefficient, $C / C_{0}$. Here, $C_{0}$ is the reference (nominal) dimensionless flow coefficient of the orifice of an electro-pneumatic transducer.

Altogether, 18 conclusions were formulated, the majority of which take a practical form of the engineering rules.

The problem of efficiency during the energy conversion of a single-action actuator was also studied and the maximum theoretical efficiency of the process was analytically determined.

The analytical model of a single-action pneumatic actuator was verified experimentally and through simulations. The conformity of the analytical model with the experiment findings for two different actuators was confirmed.

Altogether, six working hypotheses regarding the root cause of the asymmetry were formulated based on unverified expert knowledge and common perspectives. During the course of the simulation, most of the hypotheses were definitively rejected. Finally, the hypothesis explaining that the asymmetry comes as an effect of the difference in the air transformation depending on whether the chamber is charged or discharged was confirmed.

Funding: This research was funded within the Open Science model of the Excellence Initiative Program - Research University of the Warsaw University of Technology.

Conflicts of Interest: The author declare no conflict of interest. 


\section{Appendix Set of parameters used for simulations}

Table A1. Set of main parameters of investigated actuators.

\begin{tabular}{|c|c|c|c|c|}
\hline Item & Symb. & Value & Unit & Parameter \\
\hline \multicolumn{5}{|c|}{ Basic physical parameters } \\
\hline 1 & $R$ & 8.31446 & $\mathrm{Nm} /(\mathrm{Kmol})$ & gas constant \\
\hline 2 & $\delta$ & 0.02896 & $\mathrm{~kg} / \mathrm{mol}$ & air mol density \\
\hline 3 & $P_{0}$ & 101325 & $\mathrm{~Pa}$ & absolute air pressure \\
\hline 4 & $\gamma$ & 1.4 & - & heat capacity ratio \\
\hline 5 & $T_{a}$ & 298.15 & K & abs. ambient temperature \\
\hline \multicolumn{5}{|c|}{ Actuator R1-400 } \\
\hline 6 & $V$ & 0.800 & $d m^{3}$ & nominal volume \\
\hline 7 & $V_{0}$ & 0.002 & $d m^{3}$ & initial volume \\
\hline 8 & $A$ & 400 & $\mathrm{~cm}^{2}$ & effective diaphragm area \\
\hline 9 & $x_{0}$ & 10 & $m m$ & abs. initial stem travel \\
\hline 10 & $x_{b n}$ & 20 & $\mathrm{~mm}$ & nominal stem travel bench \\
\hline 11 & $p_{s}$ & 400 & $k P a$ & supply pressure \\
\hline 12 & $p_{b n}$ & $800-240$ & $k P a$ & nominal pressure bench set \\
\hline 13 & $k$ & 400 & $k N / m$ & spring constant \\
\hline \multicolumn{5}{|c|}{ Actuator $37-13$} \\
\hline 14 & $V$ & 2.581 & $d m^{3}$ & nominal volume \\
\hline 15 & $V_{0}$ & 0.004 & $d m^{3}$ & initial volume \\
\hline 16 & $A$ & 677 & $\mathrm{~cm}^{2}$ & effective diaphragm area \\
\hline 17 & $x_{0}$ & 7.62 & $\mathrm{~mm}$ & initial abs. stem position \\
\hline 18 & $x_{b n}$ & 38.1 & $\mathrm{~mm}$ & nominal stem travel bench \\
\hline 19 & $p_{S}$ & 140 & $k P a$ & supply pressure \\
\hline 20 & $p_{b n}$ & $20-100$ & $k P a$ & nominal pressure bench set \\
\hline 21 & $k$ & 120 & $k N / m$ & spring constant \\
\hline \multicolumn{5}{|c|}{ Arbitrary chosen parameters } \\
\hline 22 & $m$ & 2.0 & $k g$ & lumped mass load \\
\hline 23 & $F$ & 480 & $N$ & static friction force \\
\hline 24 & $k_{f}$ & 500 & Ns $/ m$ & viscous friction coefficient \\
\hline 25 & $C \cdot a$ & 0.893 & $m m^{2}$ & actuator R1-400 \\
\hline 26 & $C \cdot a$ & 2.177 & $m m^{2}$ & actuator $37-13$ \\
\hline 27 & $\lambda$ & 1000 & $N s / m$ & stem damping coefficient \\
\hline
\end{tabular}

\section{References}

1. Kilian, C. Modern Control Technology; Thompson Delmar Learning, 2005. ISBN: 978-1-4018-5806-3.

2. $\quad$ Emerson. Control Valve Handbook, 5 ed.; 2017; pp. 1-319.

3. Emerson. Instruction Manual Fisher 657 Diaphragm Actuator Sizes 30/30i through 70/70i and 87. Technical report, Emerson, 2018. D100306X012.

4. Masoneilan. 37/38 Pneumatic Spring Diaphragm Actuators. Technical Report GEA19531, General Electric Company Oil and Gas, 2013.

5. Polna. Diaphragm multi-spring pneumatic actuators type P1/R1. Technical Report Revision: P1/R1/06/2013, Polna S.A., 2013. available from www.polna.com.pl at May 16, 2019.

6. Samson. Type 3271 and Type 3277 Pneumatic Actuators. Mounting and Operating Instructions. Technical Report EB 8310-6 EN, Samson A.G., 2017.

7. Blagojevic, V.; Seslija, D.; Dudic, S.; Randjelovic, S. Energy Efficiency of Pneumatic Cylinder Control with Different Levels of Compressed Air Pressure and Clamping Cartridge. Energies 2020, 13. 
8. Tressler, J.M.; Clement, T.; Kazerooni, H.; Lim, M. Dynamic Behavior of Pneumatic Systems for Lower Extremity Extenders. Proceedings of the 2002 IEEE. International Conference on Robotics and Automation, 2002, pp. 3248-3253.

9. Qunli, S.; Shan'en, Y.; Haiyan, W. Modeling and experiment of pneumatic position system with spring-and-diaphragm actuator. 27th Chinese Control Conference, IEEE, $2008 . \quad$ DOI: 10.1109/CHICC.2008.4605715.

10. Bensch, U.; Helmer, J.; Kiel, B.; Rosendahl, H. Pneumatic vehicle brake system and control method. Patent: US8708430B2, 2014.

11. Huang, J.; Cao, Y.; Xiong, C.; Zhang, H. An Echo State Gaussian Process-Based Nonlinear Model Predictive Control for Pneumatic Muscle Actuators. IEEE Transactions on Automation Science and Engineering. 2018, pp. 1-14. DOI: 10.1109/TASE.2018.2867939.

12. Nguyen, T.; Leavitt, J.; Jabbari, F.; Bobrow, J.E. Accurate Sliding-Mode Control of Pneumatic Systems Using Low-Cost Solenoid Valves. IEEE/ASME Transactions on Mechatronics 2007, 12, 216-219.

13. Yu, C.C. Autotuning of PID Controllers: A Relay Feedback Approach; Springer Science \& Business Media, 2006.

14. Berner, J.; Soltesz, K.; Hägglund, T.; Äström, K.J. An experimental comparison of PID autotuners. Control Engineering Practice 2018, 73, 124-133.

15. Dihovicni, D.; Nedic, N. Simulation, animation and program support for a high performance pneumatic force actuator system. Mathematical and Computer Modelling 2008, 48, 761-768.

16. Rao, Z.; Bone, G. Nonlinear Modeling and Control of Servo Pneumatic Actuators. IEEE Transactions on Control Systems Technology 2008, 16, 562-569.

17. Olsson, H.; Astrom, K.J.; de Wit, C.C.; Gafvert, M.; Lischinsky, P. Friction models and friction compensation. European Journal of Control 1998, 4, 176-195.

18. Mehmood, A.; Laghrouche, S.; Bagdouri, M.E. Position Tracking of the VGT Single Acting Pneumatic Actuator with 2nd order SMC and Backstepping Control Techniques. 12th IEEE Workshop on Variable Structure Systems VSS12, 2012, pp. 94-99. 